\title{
An MRI Compatible Visual Force-Feedback System for the Study of Force Control Mechanics
}

\author{
Wensheng Hou ${ }^{1,2}$, Shan Shen ${ }^{2}$, Sterr, A $^{2}$. \\ ${ }^{1}$ Biomedical Department of Chongqing University, 174 Shazheng Street, Chongqing 400044, China \\ ${ }^{2}$ Psychology Department, School of Human Science, University of Surrey, Guildford, Surrey, Gu2 7XH, UK
}

\begin{abstract}
Motor task experiments play an essential role in exploring the brain mechanisms of movement control, and visual force-feedback is an important factor in these motor experiments. In this paper, the authors proposed a visual forcefeedback system suitable for neuroscience experiment. With this system, the force output produced by participants can be detected and recorded in real time, while force output was visually displayed as a feedback cue to the participants simultaneously. Furthermore, this force feedback system is MRI compatible, and can be used both in fMRI and ERP experiments. The proposed system has been applied in handgrip tasks and finger movement experiments, which were designed to explore the relationship between force output and brain activation mode in normal subject and stroke patient. The results demonstrated that various force levels were well detected and visual feedback signals enabled the accomplishment of experiments with both fixed and variable target force levels.
\end{abstract}

Index Terms-Force feedback, motor function, signal detecting, force sensor

\section{INTRODUCTION}

Motor task experiments play an essential role in the attempt to understand how the brain controls movement. Usually, these experiments are designed to detect the activation within the brain with fMRI, PET, ERP and other brain mapping methods whilst the subject performs a specific motor task.

Force feedback refers to a method whereby when a subject conducts a motor task, while force-related parameters are measured and fedback to participants by visual/audio cues. With these force feedback methods, the subject knows what their performance in the task, and can adjust their behavior to the pre-specified requirement. Force feedback has been widely used in motor experiments. Hamzei et al [3] employed auditory force-feedback in an index-thumb pincher grip task, where different sounds were given to indicate the force levels produced by the participants. Ehrsson et al [4] used a tactile cue to inform the subjects that the force is at the target level. However, the auditory feedback is not suitable for fMRI experiment, where the participants stay in intrinsicly noisy environment. Furthermore, both tactile and auditory feedback can not easily provide continuous and precise force level feedback, which is important to access the participants' force output. Therefore, visual force-feedback is the most popular method, as it can not only indicate whether the target is achieved, but also how close the force produced is to the target force. This makes it much easier for subjects to adjust their performance. Many researchers have utilized visual force-feedback in motor tasks, such as the isometric finger force production task by Slobounov et al [1], the handgrip task by Ward et al [2], and the visuomotor coordination task by Koeneke et al [5].

Different protocols of a therapeutic approach to rehabilitation of movement after stroke, are currently tested by the Clinical Neuroscience Team, University of Surrey. To study the clinical benefits and neural correlates of treatment success in stroke, hand motor experiments are employed to explore the relationship between force output and brain activation mode, using functional MRI (fMRI). Therefore, a specific force feedback system is required to detect and record the force level produced by the patients, while a visual feedback signal is generated to inform the patients whether the tasks are performed correctly. In this paper, an economic MR compatible visual force-feedback system is proposed, which is also suitable for a general application in motor experiments..

\section{BASIC CONCEPTS OF FEEDBACK IN MOTOR EXPERIMENTS}

Motor behaviors can occur along a continuum of control, ranging from feedback to feedforward. Feedback control involves modification of ongoing movement using exteroceptive information, which includes tactile and visual stimulation. Electrocortical (EEG) studies have revealed that, with visual feedback, the magnitude of force and controlsignal gain can influence the activation across two regions, the parietal lobe and the supplimentary and mesial premotor area [6], whereas posterior parietal cortex plays a critical role in the nonvisual feedback loops [13] [14].

In the literature, a variety of movement factors have been identified which correlate with the degree of activation in the brain, such as force, amplitude and frequency. Therefore, they are always considered in the design of motor experiments. The typical motor paradigms include flexion and extension of index fingers [7] [8], finger opposition movements [9], finger tapping [10], power grip [4], and rotational arm movements [12]. For example, in the power grip task, subjects are required to meet the desired force level in a given time frame. Usually, the design of a specific motor 
experiment with any of these well-known tasks needs a force-feedback method.

In a typical motor experiment using force-feedback, a subject is required to execute a specific motor task according to a visual/auditory cue, which is pre-specified in the study paradigm. The subject's brain activation is detected by coregistration of the brain response, e.g. by EEG, PET and fMRI. Additionally, selected behavior parameters are collected. These parameters can be used to reinforce the subject conducting a required task with a visual/audio feedback stimulus in real time. Furthermore, off-line analysis of these parameters can also provide helpful insight into force mechanisms (e.g. learning), as well as guiding the analysis of the imaging data, for example the separate analysis of correct/incorrect trials. Although some behavior parameters can be monitored by video camera, the precise performance of participant (such as force level, movement frequency) cannot be measured by video signals. Therefore, it is necessary to use a force feedback system to monitor the force output and record the motor task performance in real time, and with a high sampling rate. The outline of a typical motor task experiment is illustrated in Fig. 1.

\section{TECHNICAL DETAILS OF THE FORCE FEEDBACK SYSTEM}

With the requirements of motor function experiments discussed above, a visual force-feedback system was developed. This consist of a hardware system that continuously monitors the force output and a software for online signal analysis and feedback signal presentation.

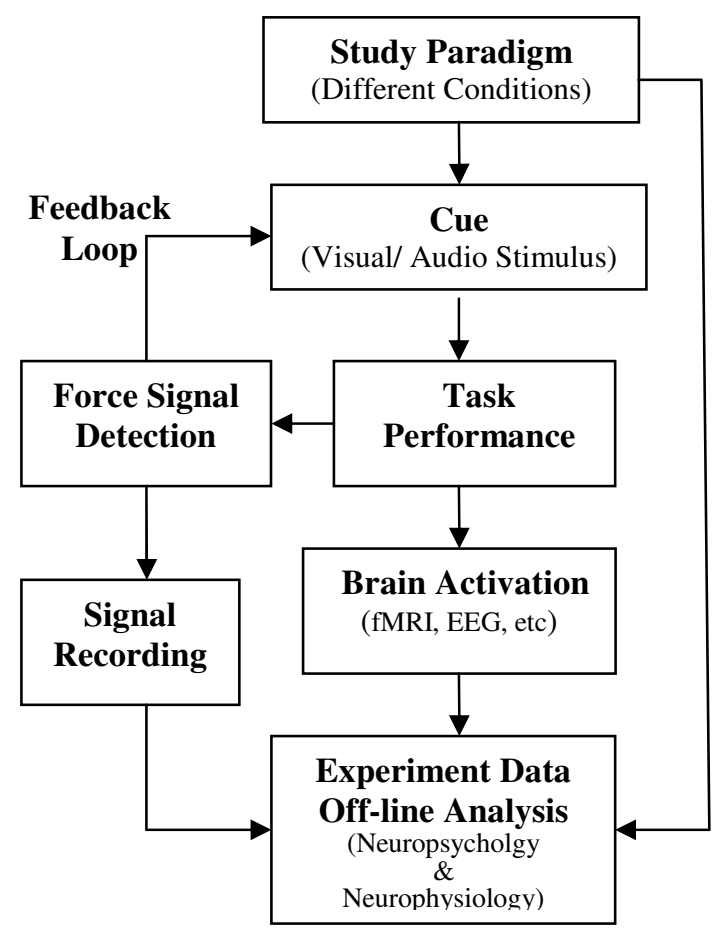

Fig. 1. A typical motor task experiment block in neuropsychology and neurophysiology research

\section{A. Force Signal Detection System}

The hardware used is a standard force signal detecting system, which includes the force sensors, an amplifier, an $\mathrm{A} / \mathrm{D}$ converter, an interface port and a PC, as illustrated in Fig. 2.

Force Sensor: For our experimental requirements, we select 4 FS serial sensors (made by HoneyWell) which measure the force signal produced by the hand or fingers. These sensors utilize a specialized piezoresistive micromachined silicon sensing element, which translates the force level to a differential voltage output.

Interface card: In order to meet the fast processing requirements of the hardware system, we also employed a commercial multi-function DAQ card (ADLINK Co.), PCI9112. This PCI card has a 12-bit A/D resolution and up to $110 \mathrm{kS} / \mathrm{s}$ sampling rate, can provide $16-\mathrm{CH}$ single-ended or 8-CH differential analog signal inputs, and has multi-level programmable gains. Also, the differential output of the force sensor can be connected to the differential input port directly, and the card can provide power for the force sensors.

PC: The main function of the PC is to process the detected force signal, calculate selected force parameters (such as movement frequency, force level), and display a variety of feedback cues in real time. Furthermore, this step must be simultaneous with the monitoring of imaging data.

Software: MATLAB (MathWorks, Inc.) is used for data acquisition and Presentation (Neurobehavioral Systems, Inc.) implements the visual stimulus and visual feedback.

For MR compatibility, the force sensors were embedded in a wood bar, and connected to the input port of PCI-9112 card with an electric-magnetic shielded cable.

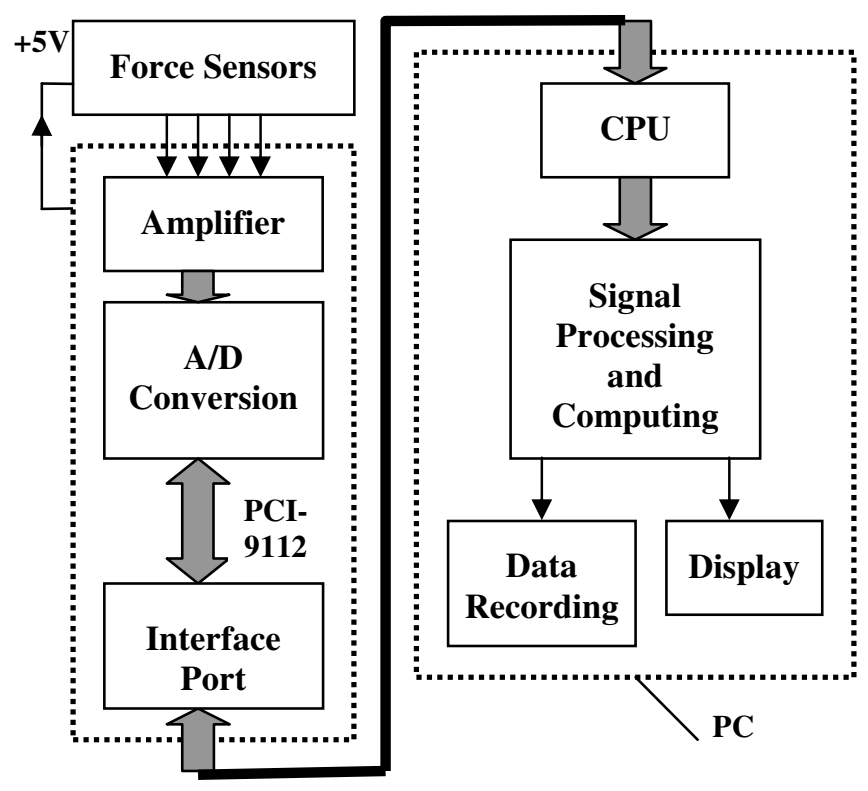

Fig. 2 Main function blocks of the constructed force feedback system 


\section{B. Feedback Procedure}

After a new trial begins, the feedback system first detects the force signal as measured by the force sensor. Then, force parameters, such as the force level and movement frequency, are calculated and compared to the target force level in a feedback loop. If the detected results match the pre-specified parameter, a message stating that the task is fulfilled is displayed; otherwise an error message is shown, reminding the subjects that their performance needs to be adjusted. This comparative feedback process continues until the task is performed correctly or the end of trial is reached. A flowchart of the hand-grip motor experiment is shown in Fig. 3. The pre-specific force target range is displayed as a horizontal bar, while the output force level is displayed as a vertical bar. The vertical bar increases and decreases in size dynamically with the increased and decreased force level. The subjects will increase their force output if the force level is too low to fit the target force range, whereas the subjects would reduce their force output if the force level is too high. The goal is to keep the force output within the target range, and that means the vertical bar must reached the horizontal bar.

\section{APPLICATIONS TO MOTOR EXPERIMENTS}

\section{A. Hand grip task with fixed target force level}

In this experiment, the subject is required to grip a

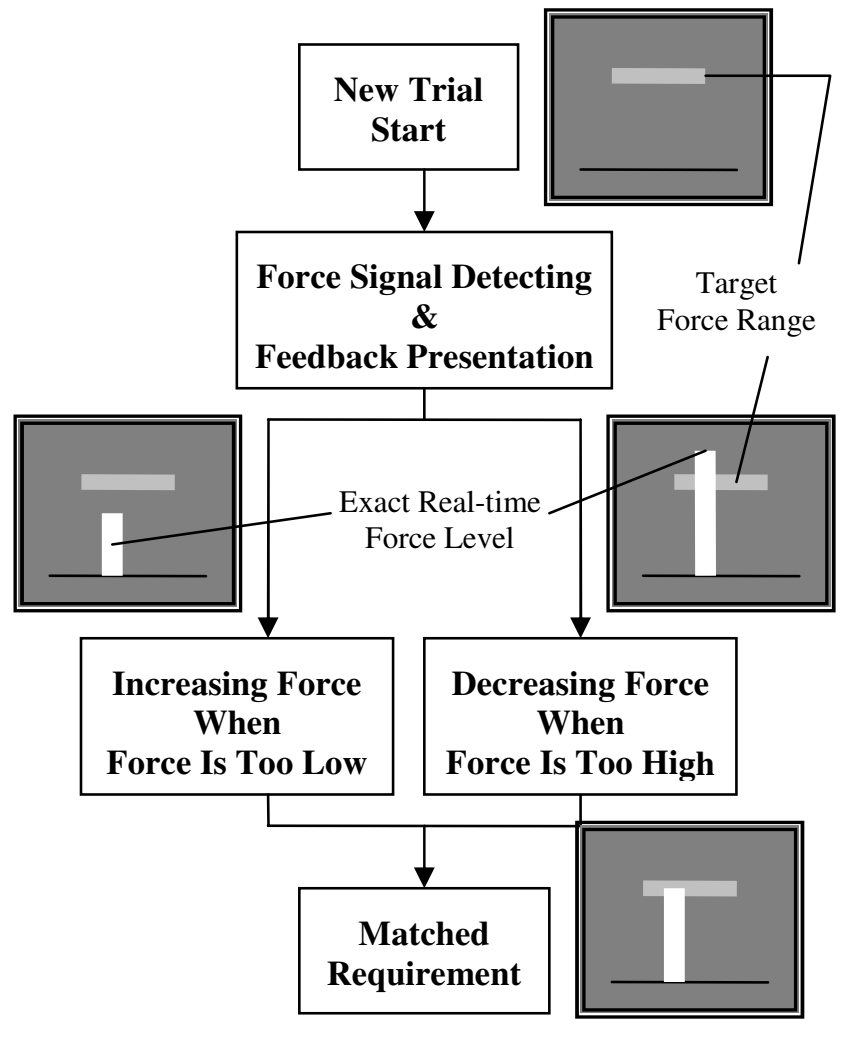

Fig.3 Flowchart of the constructed force feedback system custom-made bar with their affected hand, and produce a force level output to a pre-defined target force level. The force produced by hand is measured by force sensors embedded in the bar. Initially, Maximum Voluntary Contraction (MVC) of each participant is tested in five measurements in the experiment. The target force levels are specified at $10 \%, 40 \%$ and $70 \%$ of MVC.

The pre-specified target force level is presented as a horizontal line on the screen. The subject is asked to grip the bar as quickly as possible. Once the force output has reached the target force level, the visual cue disappears, and the subject releases their hand and waits for the next trial.

A typical time-force curve for the hand-grip experiment is illustrated in Fig. 4. The target force level is $70 \%$ of MVC.

\section{B. Finger movement task with variable target force level}

Another application of the force feedback system is the finger movement task. Subjects are required to press a custom-designed button with right index or left index finger, the force of which is measured by force sensor fixed under the button. However, the target force range is not fixed, but variable. That is to say, a specific horizontal bar which stands for target force range will keep moving dynamically on the screen while the subject executes a button-press motor task. In any one trial, the width of this bar is fixed, but it keeps moving up/down, and the subject is required to increase/decrease their force to track the target and control their force output within the target level. Furthermore, the symbol of a target force range will not move with a constant speed. When a subject can control their force output within target force range more accurately, the target force rang will move more quickly; when subject can not control their force output accurately, the target force range will move slowly. In different trials, the target bar may move with different speeds and with a different width. This allows us to create various difficulty levels for one set of effectors. A typical time-force curve for this experiment was shown in Fig. 5.

As illustrated in Fig. 4 and Fig. 5, the force output of both motor tasks can be detected in real time.

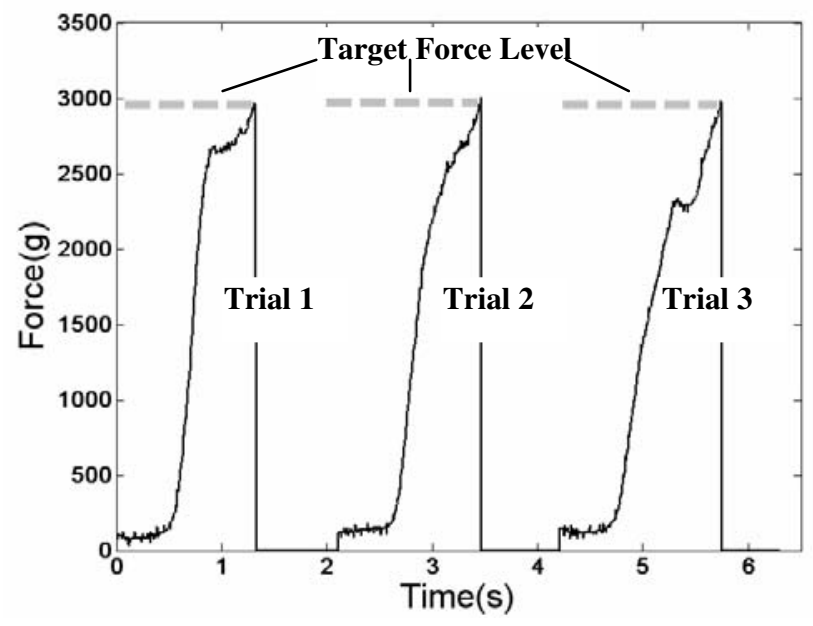

Fig. 4. Force output of hand grip task 


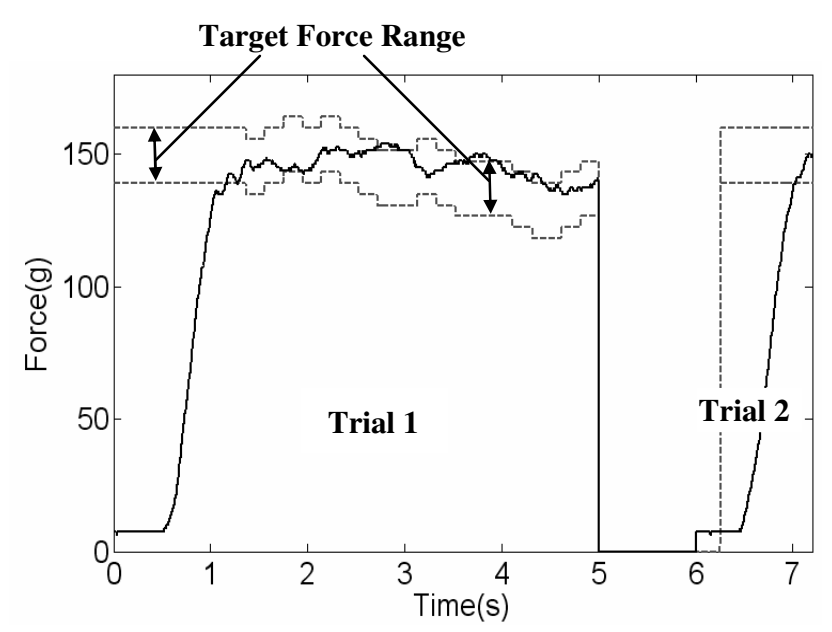

(a)

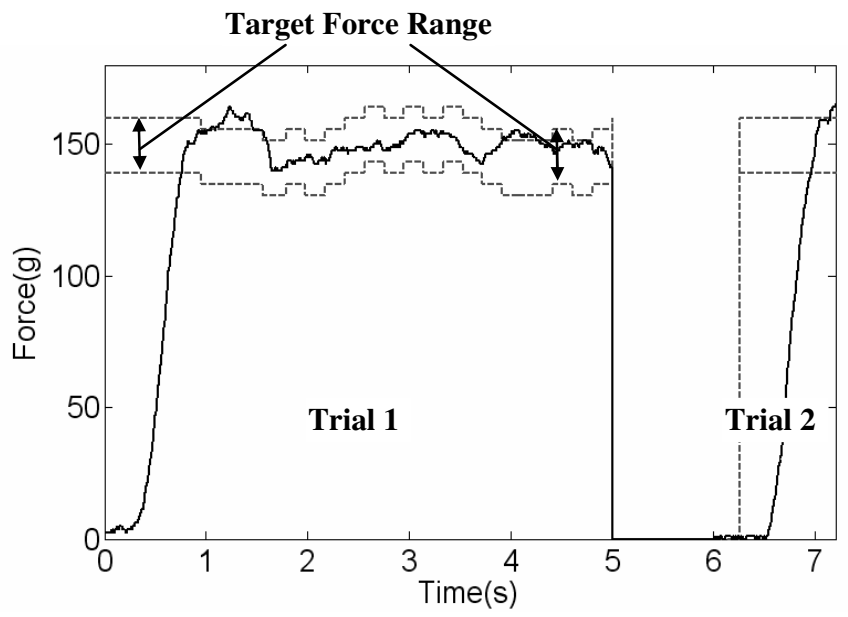

(b)

Fig. 5. Force output of index finger press task with demand of random varied target force range. (a) left index finger, (b) right index finger

\section{SUMMARY}

Motor experiments play an important role in exploring the mechanisms of brain motor control, in which force feedback is a key factor. In this paper, we introduced a force feedback system designed for motor experiments. Force outputs produced by subjects can be detected and recorded in real time using this system, and these outputs are visually displayed as a feedback cue to the subject simultaneously. Furthermore, the force feedback system is MRI compatible, and can be used in both fMRI and ERP motor experiment. It is designed to allow greatest flexibility with regards to the task difficulty level.

The described system has been tested in a typical handgrip experiment and a finger movement experiment. The behavioral results demonstrate that the described force feedback system is suitable for a variety of motor experiments in neuropsychology and neuroscience research. It is easy adaptable for other requirements.

\section{REFERENCE}

[1] S. Slobounov, J. Johnston, H. Chiang, W.J. Ray. Motor-related cortical potentials accompanying enslaving effect in singleversus combination of fingers force production tasks. Clinical Neurophysiology. Vol. 113, pp. 1444-1453, 2002

[2] N. S. Ward, M. M. Brown, A. J. Thompson, and R. S. J. Frackowiak Neural correlates of outcome after stroke: a cross-sectional fMRI study. Brain. Vol. 126, pp.1430-1448, 2003

[3] Farsin Hamzei, Christian Dettmers, Reinhard Rzanny, Joachim Liepert. Reduction of Excitability ("Inhibition") in the Ipsilateral Primary Motor Cortex Is Mirrored by fMRI Signal Decreases. NeuroImage. Vol. 17, pp. 490-496, 2002

[4] Ehrsson, H. Henrik, Anders Fagergren, Tomas Jonsson, Goran Westling, Roland S. Johansson, and Hans Forssberg. Cortical activity in precision- versus power-grip tasks: an fMRI study. J. Neurophysiol. Vol. 83, pp. 528-536, 2000.

[5] Susan Koeneke, Kai Lutz, Torsten Wustenberg, and Lutz Jancke. Bimanual versus unimanual coordination: what makes the difference? NeuroImage 22 (2004) 1336- 1350

[6] 8 Matthew P. Rearick, Jamie A. Johnston, Semyon M. Slobounov. Feedback-dependent modulation of isometric force control: an EEG study in visuomotor integration. Cognitive Brain Research. Vol. 12, pp. 117-130, 2001

[7] Immisch, I., Waldvogel, D., van Gelderen, P., Hallett. The role ofthe medial wall and its anatomical variations for bimanual antiphase and in-phase movements. NeuroImage. Vol. 14, pp. 674-684, 2001.

[8] Sadato, N., Yonekura, Y., Waki, A., Yamada, H., Ishii, Y. Role of the supplementary motor area and the right premotor cortex in the coordination of bimanual finger movements. J. Neurosci. Vol. 17, pp. 9667-9674, 1997.

[9] Stephan, K.M., Binkofski, F., Posse, S., Seitz, R.J., Freund, H.J., 1999b. Cerebral midline structures in bimanual coordination. Exp. Brain Res. Vol. 128b, pp. 243-249, 1999.

[10] Jancke, L., Peters, M., Himmelbach, M., Nosselt, T., Shah, J., Steinmetz, H. fMRI study of bimanual coordination. Neuropsychologia. Vol. 38b, pp. 164-174, 2000.

[11] Tracy, J.I., Faro, S.S., Mohammed, F.B., Pinus, A.B., Madi, S.M., Laskas, J.W. Cerebellar mediation of the complexity of bimanual compared to unimanual movements. Neurology. Vol.57, pp. 1862 1869, 2001.

[12] Viviani, P., Perani, D., Grassi, F., Bettinardi, V., Fazio, F. Hemispheric asymmetries and bimanual asynchrony in left- and righthanders. Exp. Brain Res. Vol. 120, pp. 531- 536, 1998.

[13] S.M. Slobounov, K. Fukada, R. Simon, M. Rearick, W. Ray, Neurophysiological and behavioral indices of time pressure effects on visuomotor task performance. Cognit. Brain Res. Vol. 9, pp. 287-298, 2000

[14] M.E. Smith, L.K. McEvoy, A. Gevins, Neurophysiological indices of strategy development and skill acquisition. Cognit. Brain Res. Vol. 7, pp. 389-404, 1999. 